\title{
Ferromagnetism and orbital order in a topological ferroelectric
}

\author{
Marco Scarrozza, Alessio Filippetti, and Vincenzo Fiorentini \\ CNR-IOM, UOS Cagliari, and Department of Physics, University of Cagliari, \\ Cittadella Universitaria, I-09042 Monserrato (CA), Italy
}

(Dated: October 25, 2018)

\begin{abstract}
We explore via density functional calculations the magnetic doping of a topological ferroelectric as an unconventional route to multiferroicity. Vanadium doping of the layered perovskite $\mathrm{La}_{2} \mathrm{Ti}_{2} \mathrm{O}_{7}$ largely preserves electric polarization and produces robust ferromagnetic order, hence proper multiferroicity. The marked tendency of dopants to cluster into chains results in an insulating character at generic doping. Ferromagnetism stems from the symmetry breaking of the multi-orbital V system via an unusual "antiferro"-orbital order, and from the host's low-symmetry layered structure.
\end{abstract}

PACS numbers: 75.85.+t,77.84.-s,75.30.-m,71.15.Mb

Multiferroic materials, where ferroelectricity and magnetism coexist, have been studied intensively in recent years [1] due to their fascinating physical properties and their potential for application (e.g. multi-state or electrically switchable magnetic memories) 2]. Despite the effort, single-phase bulk multiferroics at room temperature are still unavailable; most are antiferromagnetic ( $\mathrm{AF}$ ) and not, as desired, ferromagnetic (FM); and, generally, they do not meet 3 the integration and functional requirements of device applications [4, 5]. A parallel line of research [3, 6] on multi-phase systems (interfaces, heterostructures, nanostructures) revealed unconventional ferroelectricity mechanisms in artificially layered perovskites (e.g. $\mathrm{SrTiO}_{3} / \mathrm{PbTiO}_{3}$ superlattices [7). Recently, it was suggested [8, 9] that magnetism and ferroelectricity will be more cooperative in lower-symmetry, naturally nano-structured, layered systems. In one such system, the layered perovskite oxide $\mathrm{La}_{2} \mathrm{Ti}_{2} \mathrm{O}_{7}$ (LTO), ferroelectricity is not displacive but rather results from dipoles generated by antiferrodistortive oxygen-octahedra rotations, which fail to cancel out due to the layered structure, resulting in a net macroscopic polarization [9]. Because ferroelectricity follows directly from its layered structure, LTO was labeled [9] a topological ferroelectric. LTO has a $\mathrm{T}_{\mathrm{C}}$ of $1770 \mathrm{~K}$, a sizable polarization $P_{c}=5 \mu \mathrm{C} / \mathrm{cm}^{2}$ [10] along the $c$ crystal axis (schematic in Fig.2), and non-critical permittivity $\varepsilon \simeq 50$ which would be an asset for integration in ferroelectric random-access memory elements [1].

Here we explore theoretically a route to multiferroicity based on doping LTO with magnetically active ions substituting for $\mathrm{Ti}$ (nominally a $4+3 d^{0}$ ion). By an extensive screening of transition-metal dopants, we found vanadium $\left(\mathrm{V}\right.$, nominally $4+$ and $3 d^{1}$ in LTO) to yield robust ferromagnetic order while preserving polarization and a small gap, thus giving rise to proper multiferroicity. We find that $\mathrm{V}$ align in chains along the $a$ axis, orthogonal to the polarization $c$ axis (with magnetic moments orthogonal to the chains themselves), resulting in insulating character at generic doping. Intriguingly, ferromagnetic coupling is linked to the layered structure. The latter "pre-wires" a two-orbital-per-site system, whose degeneracy is broken in favor of ferromagnetic superexchange by a peculiar orbital ordering, as foreshadowed in earlier theoretical work [12. We preliminarily investigate the magnetoelectric response to (a subset of) ionic vibrations, finding it to be almost absent.

We perform ab initio calculations within spin-density functional theory (DFT) in the generalized gradient approximation (GGA) 13] as implemented in the VASP code [14, with projector-augmented waves 15] for La $5 s p d 6 s$, Ti and $\mathrm{V} 3 p d 4 s$, and $\mathrm{O} 2 s p$ states, plane-wave cutoff $330 \mathrm{eV}$, and a $4 \times 2 \times 5$ grid in the primitive cell's Brillouin zone, folded for larger supercells. Magnetic calculations for magnetoelectricity and anisotropy are non-collinear and include spin-orbit coupling (see below). GGA seems a reliable approach for LTO, which is a band insulator with $\mathrm{O} p$ valence and Ti $d$ conduction [9]; even the $\mathrm{V}$ electrons (see below) sit into conduction-derived states, whose the localization is considerable but not extreme.

LTO is the $n=4$ member of the $\mathrm{A}_{n} \mathrm{~B}_{n} \mathrm{O}_{3 n+2}$ family, and consists of four perovskite-like units (distorted $\mathrm{TiO}_{6}$ octahedra) stacked along the [011] cartesian direction and separated periodically by the insertion of an extra O layer [16. We optimize cell and internal parameters (force threshold $20 \mathrm{meV} / \AA$ ) in the experimentally-stable monoclinic $P 2_{1}$ structure. Our calculated lattice parameters $a=7.80 \AA, b=13.22 \AA, c=5.58 \AA$, and $\gamma=98.52^{\circ}$ are in excellent agreement with experiment [17. The energetics and electronic structure of V-doped LTO is obtained collating data from calculations on 44-atom and 88 -atom cells at $12.5 \%$ average $\mathrm{V}$ concentration. Our conclusions do not really depend critically on this figure, which in essence only amounts to approximate the isolated-impurity limit with a single $\mathrm{V}$ at no less than about $8 \AA$ from its periodic image. The isolated V's spinpolarized electron yields a magnetic moment of $1.0 \mu_{B}$. Its spin-resolved and atom-projected density of states (DOS) shown in Fig 1 is typical of all FM configurations of $\mathrm{V}$ discussed below. The valence band is mostly of $\mathrm{O} p$ character, and the conduction band is mostly of 
O-hybridized Ti $d$ character. Near the Fermi level, V induces two spin-majority peaks, one carrying the extra electron and the other empty, across a gap of $0.3 \mathrm{eV}$. Ti states start $0.5 \mathrm{eV}$, and minority $\mathrm{V} d$ states at $\sim 0.85 \mathrm{eV}$ above the edge of the occupied $\mathrm{V}$ band.

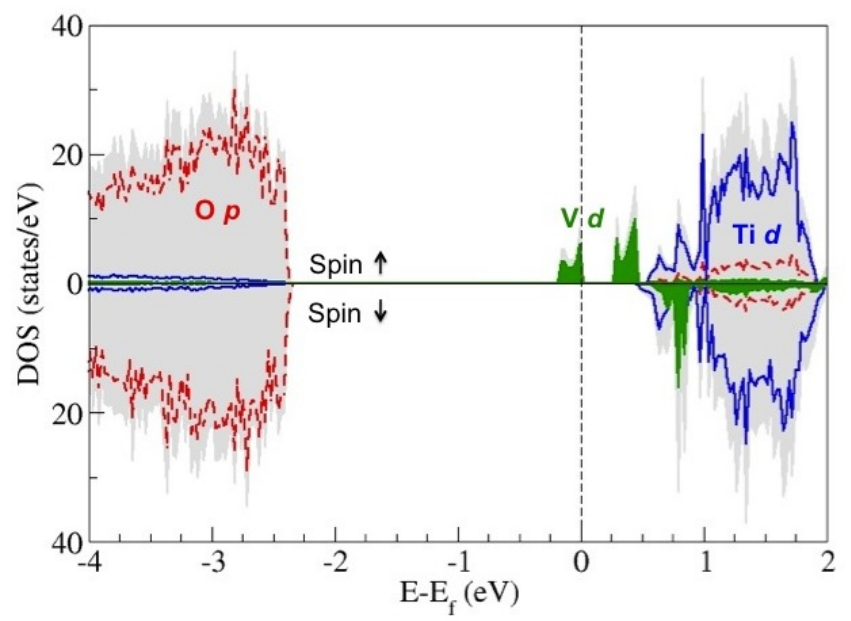

FIG. 1. Total density of states (DOS) of LTO:V (gray shade) and its site projections for $\mathrm{O}$ (dashed red line), Ti (solid blue line), and V (green shade).

To estimate the magnetic coupling between V's, we sampled a variety of structural ( $\mathrm{V}$ pairs on the relevant non symmetry-equivalent cation $\mathrm{B}$ sites) and magnetic (collinear FM and AF) configurations. Structurally, we find a tendency to chain ordering of $\mathrm{V}$ along the $a$ direction (see Fig 2). Chains are favored by $100 \mathrm{meV}$ per $\mathrm{V}$ pair over the first competing configuration, and 180 $\mathrm{meV}$ per $\mathrm{V}$ pair referred to isolated impurities. Magnetically, FM order is very stable within the $\mathrm{V}$ chains, with FM couplings $J_{a} \simeq 80 \mathrm{meV}$ and $20 \mathrm{meV}$ along the $a$ direction, respectively, for chains in proximity of the extra $\mathrm{O}$ layer or inside the perovskite block. Both types of chain (or fragment thereof, see below) will appear in similar concentrations as their energies differ by only about 5 $\mathrm{meV} /$ dopant. The interchain coupling in the $b, c$ plane is also FM and small, $J_{b, c} \simeq 3 \mathrm{meV}$ at $12 \% \mathrm{~V}$.

For a ballpark estimate of the critical temperature we use the Ising model with axial anisotropy [18, whose critical temperature $\mathrm{T}_{\mathrm{C}}$ is determined by the ratio $R=J_{b, c} / J_{a}$ of in-plane to on-axis coupling. The model mirrors closely our system of weakly coupled chains with strong internal coupling along one axis. We estimate the FM $\mathrm{T}_{\mathrm{C}}$ for both our two nearly-degenerate ground states with distinct $J_{a}$ 's, since magnetic couplings are not additive. For $J_{a} \simeq 80 \mathrm{meV}, R=0.04$ and correspondingly $\mathrm{T}_{\mathrm{C}} \sim 0.93 J_{a}=860 \mathrm{~K}$; for $J_{a} \simeq 20 \mathrm{meV}, R=0.15$ and $\mathrm{T}_{\mathrm{C}} \sim 1.55 J_{a}=360 \mathrm{~K}$, i.e. $\mathrm{T}_{\mathrm{C}}$ is around room temperature in the worst-case estimate.

We now check that the material remains ferroelectric upon doping. The polarization calculated via the Berry phase technique [19] for LTO is $P_{c}^{0}=5.2 \mu \mathrm{C} / \mathrm{cm}^{2}$ along the $c$ axis, similarly to experiment and previous calculations [10, 20. In V-doped LTO, the distortions generating ferroelectricity are fully conserved, aside from minor quantitative differences. We find a slightly enhanced and reduced $P_{c}$ for the energetically-favored chain configurations, respectively, inside the blocks and near the additional O layers. Since polarization is extensive, we average the values for these nearly-degenerate configurations obtaining $P_{c}=4.7 \mu \mathrm{C} / \mathrm{cm}^{2}$, i.e. only $10 \%$ lower than undoped LTO at $12.5 \% \mathrm{~V}$ concentration. From $P$ values at $12 \%$ and $25 \% \mathrm{~V}$, we infer that $P_{c}[x] \simeq P_{c}^{0}(1-x)$ with $x$ $\% \mathrm{~V}$. Interestingly, $\mathrm{V}$ doping produces polarization components along $a$ and $b, 4.5 \mu \mathrm{C} / \mathrm{cm}^{2}$ and $0.7 \mu \mathrm{C} / \mathrm{m}^{2}$ respectively at $12 \% \mathrm{~V}$, comparable with that along $c$. We will address the consequences of this elsewhere.

So far we saw that V-doped LTO is insulating, polarized, and ferromagnetic, and therefore properly multiferroic. To show that this applies at generic doping, we evaluate energetics, magnetism, and insulating character of $\mathrm{V}$ chain fragments in LTO comparing three configurations with 4 cations lined up along $a: i)-\mathrm{V}-\mathrm{V}-\mathrm{V}-\mathrm{V}-, i i)$ -V-V-Ti-Ti-, and iii) -V-Ti-V-Ti-. The full chain $i$ ) is bound by $100 \mathrm{meV}$ compared to two separated V dimers (chain fragments) as in ii). In turn, the $\mathrm{V}$ dimer fragment ii) is $130 \mathrm{meV}$ lower in energy than the "broken" dimer iii). Energy thus drives V to cluster in homogeneous chains; these will be in fact finite chain fragments accounting for configurational entropy (we will elaborate on this elsewhere.) All chain and chain-fragment configurations are insulating, and $i$ ) and $i i$ ) have similar FM coupling along $a$ as measured by the FM-AF energy difference; these are clear indications that the system will be insulating and FM at a generic concentration due to the $\mathrm{V}$ tendency to chain clustering.

We now analyze the strong ferromagnetic coupling. Vdoped LTO is at first glance a degenerate multi-orbital system. However, this degeneracy is resolved so as to favor FM order, and the mechanism is, to a significant extent, built-in into the host structure. First, only two orbitals per $\mathrm{V}$ site are involved: the $\mathrm{V} 3 d$ DOS of a full $\mathrm{V}$ chain in Fig 3 a shows $d_{x y}, d_{x z}$ states lower in energy and involved in V-V bonding and magnetism, while $d_{y z}$ follows the $e_{g}$ states to higher energy. Indeed, in the $2^{\prime}$ point group of LTO the octahedral-symmetry $t_{2 g}$ and $e_{g}$ representations split into 1-fold $a_{1}$ and $b_{1} . d_{y z}$ and $e_{g}$ functions belongs to $a_{1}$, whereas $d_{x y}$ and $d_{x z}$ belong to $b_{1}$. Physically, one notices that the periodic alignment of octahedra along their axes is interrupted by the layered structure, except along the $a$ axis on which $d_{x y}$ and $d_{x z}$ overlap significantly. Second, the bonding-antibonding combination (Fig 3 of $d_{x y}$ and $d_{x z}$ along the chain generates two narrow bands separated by a small gap (see e.g. 21 for a similar case). Third, because of the large energy gain due to Hund on-site exchange, FM order will clearly be favored over AF (whose much smaller gain is of 


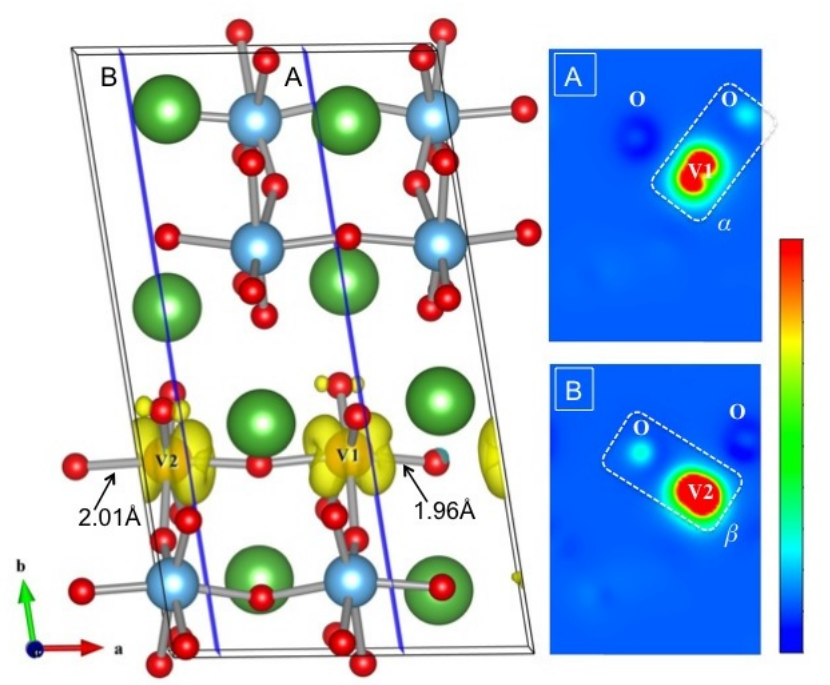

FIG. 2. On the right: Isosurface plot of the spin density for the LTO:V in V-chain configuration; on the left: 2D cuts of the spin density along the two A and B planes, normal to the $a$ axis, in proximity of the V1 and V2 atoms, respectively.

order the superexchange energy), provided that, fourth, the two-orbital bonding combination is non-degenerate, and hence different on each V site, so as to host two spinparallel electrons. Indeed, Fig 3 a clearly shows the different weight of the two orbitals in the bonding state on a specific site; this alternation is accompanied by modulated bond lengths and by differences in $\mathrm{V}$ magnetic moments, as shown below. The resulting FM spin order and "antiferro"-orbital order for the orbital filling agree with early predictions [12] as well as with GoodenoughKanamori rules 22]. The chain-like ordering of V, we note, is actually relevant even in some binary vanadates, as discussed e.g. in [23].

The computed spin density shown in Fig 2 further confirms the above argument. It is localized on $\mathrm{V}$ atoms and has the fingerprint shape of a $t_{2 g}$-like combination. The $2 \mathrm{D}$ cuts of the spin density along planes (A and B in the figure) normal to the $a$ axis and close to the $\mathrm{V}$ atoms, clearly show "antiferro"-orbital order. The spin density lobes on the two $\mathrm{V}$ sites are distinctly slanted towards alternating $\mathrm{O}$ atoms in the upper layer, one roughly normal to the other. The $\mathrm{V}$ chain has resolved the degeneracy by alternating $d_{x y}-d_{x z}$ orbital combinations $\alpha$ and $\beta$, respectively with $65 / 35 \%$ and $54 / 46 \% d_{x y} / d_{x z}$ content. As hinted-to above, concomitantly with spin and orbital ordering, the V-chain is also characterized by dimerized bond lengths and moments (see Fig.2p: pairs of short $(1.95$ and $1.97 \AA)$ and long (2.00 and $2.02 \AA)$ O-V-O bonds alternate along the chain direction, and the magnetic moments of short-bond and long-bond $\mathrm{V}$ are also alternating $\left(0.75\right.$ and $\left.1.03 \mu_{B}\right)$. As seen in Fig 2 state $\beta$ is centered on the long-bond large-moment $\mathrm{V}_{2}$ atom and $\alpha$ on $\mathrm{V}_{1}$.
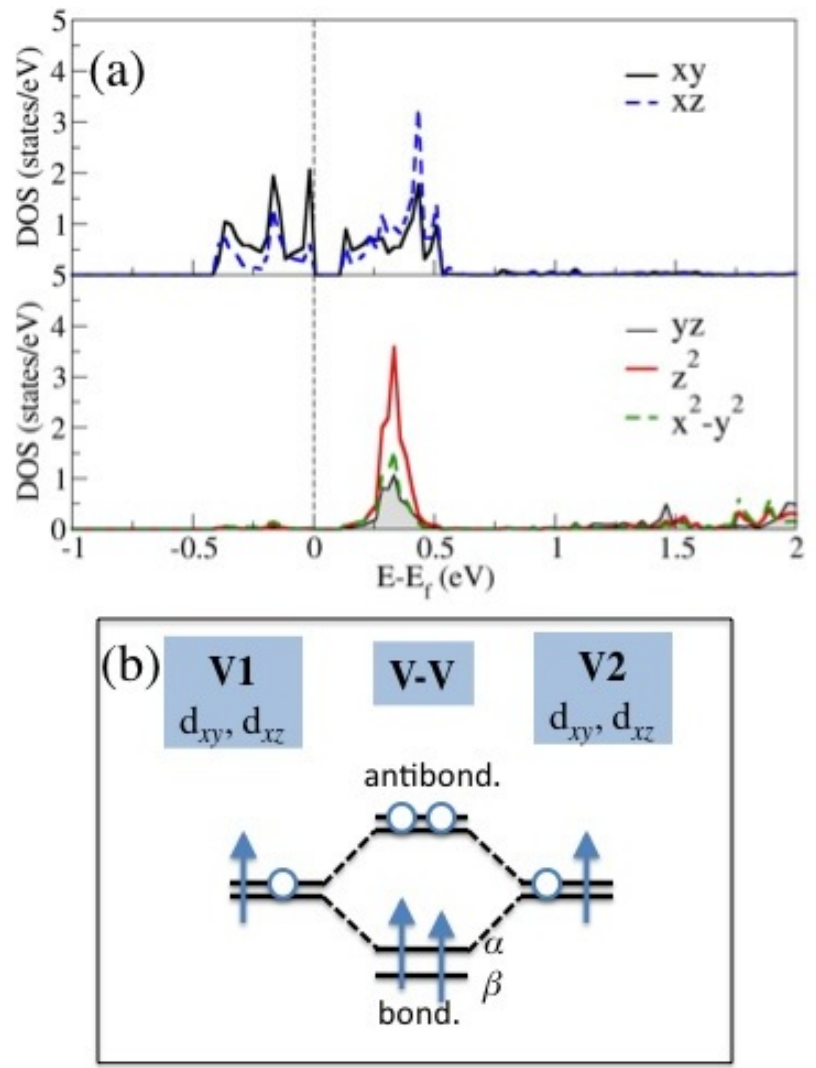

FIG. 3. (a) Projected orbital resolved DOS relative to a $\mathrm{V}$ atom in LTO:V with $2 \mathrm{~V} /$ cell, the $\mathrm{V}$-chain configuration. (b) Schematic energy levels diagram for interacting $\mathrm{V}-\mathrm{V}$ pair in LTO:V.

To further validate the link between $\mathrm{V}$ dimerization and orbital ordering, we compare V-doped LTO in $P 2_{1}$ symmetry with the parent high-symmetry structure with space group $P 2_{1} / m$. This structure has no antiferrodistortive rotations and only one inequivalent $\mathrm{V}$ site along each $a$ chain, with $\mathrm{V}-\mathrm{V}$ distances $3.91 \AA$ and $180^{\circ}$ $\mathrm{O}-\mathrm{V}-\mathrm{O}$ angle. Thus, it cannot remove the orbital degeneracy, and the bonding-antibonding splitting does not materialize. The system ends up being metallic and ferromagnetic, with identical spin-density on all $\mathrm{V}$ atoms. The structural dimerization is evidently an integral part of the explanation of the insulating nature and the spin and orbital ordering in V-doped LTO, as its absence causes orbital degeneracy and metallicity. In this very light, and in keeping with our previous symmetry considerations, we note that the O-Ti-O bond lengths along $a$ are already modulated in LTO $(1.97,2.03,1.98$, and $2.02 \AA)$. The structural inequivalencies along the $a$ axis are thus at least partly pre-wired in the host structure's low symmetry, supporting the general idea of a search for multiferroicity opportunities in unconventional, low symmetry materials.

Since multiferroicity and magnetoelectricity (the cou- 
pling of, e.g., electric field to magnetization) are often associated, we study preliminarily the lattice component of the magnetoelectric tensor, expressed by Íñiguez 24 as the product of polarization and magnetization changes in response to polar-mode distortion divided by the mode force constant. We need magnetic non-collinear spin-orbit calculations with full spin relaxation and a stricter convergence limit than usual $(<0.01 \mu \mathrm{eV})$. We consider small displacements away from the ferroelectric $P 2_{1}$ structure along the composite IR mode (mainly rotations around $a$ ) connecting it to the paraelectric $P 2_{1} / m$ structure. To numerical accuracy, all magnetization components are insensitive to this composite displacement. A rationale is provided by magnetic anisotropy calculations, whereby we constrain the spin direction to map out the energy vs spin orientation. The $\mathrm{V}$ spins preferentially point normal to the chain direction-that is, $a$ is the hard axis, with a substantial anisotropy energy 180 $\mu \mathrm{eV} /$ spin with respect to the $b, c$ plane. The $b, c$ plane, in turn, hosts many spin-configurational minima within less than $5 \mu \mathrm{eV} / \mathrm{spin}$, i.e. we all directions in the $b, c$ plane are equally easy: thus, spins "freewheel" around the $a$ axis irrespective of the rotations (we dub this the "ball-bearing" effect) and are insensitive to octahedra rotations around $a$. Interestingly, $\mathrm{V}$ spins are always approximately parallel, and they point near the [011] direction in the $b, c$ plane in the lowest-energy configuration. In conclusion, for the composite ferro-para IR mode the lattice magnetoelectric tensor is zero. This may not be the whole story, however, as we did not consider all possible IR modes; some of those we neglected may couple to the $a$ and $b$ polarization components appearing in $\mathrm{V}$-doped LTO. Also, we ignored the electronic component, which might still be appreciable.

Finally, we investigate the effect of oxygen vacancies (which are donor defects) on magnetism, motivated by our finding for Sc-doped LTO that the vacancy formation energy drops to almost zero, destroying ferromagnetism. In equilibrium oxygen-rich conditions (chemical potential $\mu_{\mathrm{O}}=\mu_{\mathrm{O}_{2}} / 2$ ), the vacancy concentration is negligible at typical growth temperatures, because the lowest (as function of configuration) formation energy of the vacancy in the neutral state in LTO is $5.4 \mathrm{eV}$. In Vdoped LTO, the concentration is still negligible, as the formation energy is $4.6 \mathrm{eV}$. Consistently with the lower formation energy, the vacancy binds to $\mathrm{V}$ pairs within the $a$ chain, turning the magnetic ordering from FM to AF. This suggests the possibility to spatially structure the magnetic state by controlling the local $\mathrm{O}$ content in layer-by-layer growth techniques.

In summary, the V-doped layered ferroelectric LTO shows several unique properties of basic as well as applicative (e.g. data storage) interest. It is a ferromagnetic multiferroic with high temperature ferroelectricity and ferromagnetism at any doping, due to $\mathrm{V}$ clustering in longish, homogeneous chains. Polarization is significant, and decreases mildly as function of $\mathrm{V}$ concentration (at least at small doping). Magnetoelectric coupling seems negligible at the present approximation level. Control of native defects could turn FM order into AF, enabling the engineering of resistivity and magnetic phase changes via defect injection and migration.

Work supported in part by IIT via Seed project NEWDFESCM, by MIUR via project 2DEG-FOXI, and by Fondazione Banco di Sardegna. Computing resources: CASPUR, CINECA, Cybersar.

[1] S. Picozzi and C. Ederer, J. Phys.: Condens. Matt. 21, 303201 (2009); C. Ederer and N. A. Spaldin, Curr. Op. Sol. State Mat. Sci. 9, 128 (2005); D. Khomskii, Physics 2, 20 (2009).

[2] W. Eerenstein, N. D. Mathur, and J. F. Scott, Nature 442, 759 (2006).

[3] L. W. Martin, S. P. Crane, Y.-H. Chu, M. B. Holcomb, M. Gajek, M. Huijben, C.-H. Yang, N. Balke, and R. Ramesh, J. Phys.: Cond. Matt. 20, 434220 (2008).

[4] H. Béa, M. Gajek, M. Bibes, and A. Barthélémy, J. Phys.: Cond. Matt. 20, 434221 (2008).

[5] C. Binek and B. Doudin, J. Phys.: Cond. Matt. 17, L39 (2005).

[6] R. Ramesh, and N. A. Spaldin, Nature Materials 6, 21 (2007).

[7] E. Bousquet, M. Dawber, N. Stucki, C. Lichtensteiger, P. Hermet, S. Gariglio, J.-M. Triscone, and P. Ghosez, Nature 452, 732 (2008).

[8] N. A. Benedek and C. J. Fennie, Phys. Rev. Lett. 106, 107204 (2011).

[9] J. López-Pérez and J. Íñiguez, Phys. Rev. B 84, 075121 (2011).

[10] S. Nanamatsu, M. Kimura, K. Doi, S. Matsushita, and N. Yamada, Ferroelectrics 8, 511 (1974).

[11] V. V. Atuchin, T. A. Gavrilova, J.-C. Grivel, and V. G. Kesler, J. Phys. D: Appl. Phys. 42, 035305 (2009).

[12] K. I. Kugel and D. I. Khomskii, Sov. Phys. Usp. 25, 231 (1982).

[13] J. P. Perdew, J. A. Chevary, S. H. Vosko, K. A. Jackson, M. R. Pederson, D. J. Singh, and C. Fiolhais, Phys. Rev. B 46, 6671 (1992).

[14] G. Kresse and J. Furthmüller, Phys. Rev. B 54, 11169 (1996); G. Kresse and D. Joubert, Phys. Rev. B 59, 1758 (1999).

[15] P. E. Blöchl, Phys. Rev. B 50, 17953 (1994).

[16] F. Lichtenberg, A. Herrnberger, K. Wiedenmann, and J. Mannhart, Prog. Sol. St. Chem. 29, 1 (2001); F. Lichtenberg, A. Herrnberger, and K. Wiedenmann, Prog. Sol. St. Chem. 36, 253 (2008).

[17] M. Gasperin, Acta Cryst. B 31, 2129 (1975).

[18] M. Yurishchev, arXiv:cond-mat/0312555.

[19] R. Resta, J. Phys.: Condens. Matter 12, R107 (2000).

[20] E. Bruyer and A. Sayede, J. Appl. Phys. 108, 053705 (2010)

[21] P. Mahadevan, A. Zunger, and D. D. Sarma, Phys. Rev. Lett. 93, 177201 (2004).

[22] J. B. Goodenough, Magnetism and the Chemical Bond (Wiley-Interscience, New York 1963); J. B. Goodenough, 
Scholarpedia 3, 7382 (2008).

[23] V. Eyert, Phys. Rev. Lett. 107, 016401 (2011); Ann. Phys. (Leipzig) 11, 650 (2002).
[24] J. Íñiguez, Phys Rev. Lett. 101, 117201 (2008). 